\title{
Acute hyperhidrosis and postural tachycardia in a COVID-19 patient
}

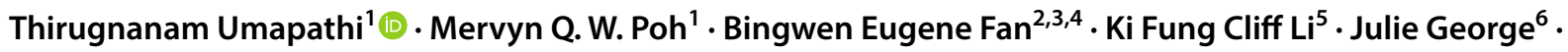 \\ Jackie Y. L. $\operatorname{Tan}^{6}$
}

Received: 21 July 2020 / Accepted: 15 September 2020 / Published online: 24 September 2020

c) Springer-Verlag GmbH Germany, part of Springer Nature 2020

Keywords Orthostatic tachycardia $\cdot$ COVID-19 $\cdot$ Dysautonomia

\section{Dear Editor,}

We describe a COVID-19 patient with acute hyperhidrosis and symptomatic orthostatic tachycardia. We encountered 3 other patients with ophthalmic dysautonomia. We posit COVID-19 as a cause of acute, limited, possibly dysimmune, autonomic dysfunction.

A 39-year-old man, a construction worker with no medical history, was diagnosed with COVID-19 from nasopharyngeal swab reverse transcription polymerase chain reaction (rt-PCR) when he presented with 8 days of acute respiratory symptoms, diarrhea, abdominal discomfort and pneumonia. Within 2 days, he required supplemental oxygen and prone-positioning, and was placed on a remdesivir trial. He recovered without ventilatory support. His blood pressure at admission was $165 / 92 \mathrm{mmHg}$. In hospital, it ranged from 130 to $170 / 80-110 \mathrm{mmHg}$. He was started on amlodipine $2.5 \mathrm{mg}$. His blood glucose ranged from 9 to $13 \mathrm{mmol} / \mathrm{L}$ and $\mathrm{HbA} 1 \mathrm{c}$ was $8.8 \%$. He was diagnosed with diabetes mellitus (DM) and given insulin and metformin.

At day 13 of illness, as he was recuperating in the general ward with stable blood pressure and parameters, he developed right leg ischemia. Computed tomography (CT) aortogram showed a mural thrombus at the suprarenal aorta.

Thirugnanam Umapathi

umapathi@nni.com.sg

1 National Neuroscience Institute, Singapore, Singapore

2 Department of Haematology, Tan Tock Seng Hospital, Singapore, Singapore

3 Lee Kong Chian School of Medicine, Singapore, Singapore

4 Yong Loo Lin School of Medicine, Singapore, Singapore

5 Department of Cardiology, Tan Tock Seng Hospital, Singapore, Singapore

6 Department of General Medicine, Tan Tock Seng Hospital, Singapore, Singapore
Aortic tributaries were unobstructed. Infarcts were limited to the spleen and upper pole of the right kidney. The adrenal gland was normal in appearance. There was no thrombosis of the vena cava. He underwent embolectomy and endovascular repair. Duration of ischemia to reperfusion was approximately $26 \mathrm{~h}$. After initial unfractionated heparin, he was given aspirin and warfarin. The raised factor VIII levels, von Willebrand factor antigen, fibrinogen, anti-cardiolipin antibodies and presence of lupus anticoagulant (Table 1) indicated a COVID-19-associated immuno-thrombotic state [1]. He was extubated the day after surgery and sent to the general ward 2 days later.

On day 18 , he complained of profound, intermittent bouts of sweating on his trunk and thighs. Simultaneously, he developed constipation, nausea and post-meal upper abdominal discomfort. He had marked symptomatic tachycardia (140/min) on standing, without associated orthostatic hypotension. However, he no longer required amlodipine. He had no headache, abnormal pilomotor activity or hypertension during these bouts. He complained of dry mouth, but not dry eyes, erectile dysfunction, or urinary or near-vision difficulty. He had mild patchy loss of light touch, pain and vibration sense, mainly in the ischemic toes of the right leg. His fingers were not numb. He had no signs of ischemic myeloradiculopathy. His pupils reacted well to light and near stimulus, and near vision was normal. Hormonal evaluation showed increased urinary catecholamines (Table 1). Autonomic function tests (Table 1), delayed to day 42 of illness by infection control restrictions, showed marked tachycardia on standing (70-105/min after $3 \mathrm{~min}$ ) and during passive 60-degree tilt (67-98/min after $5 \mathrm{~min}$ ) without decrease in blood pressure. The electrocardiogram showed a negative delta wave in inferior leads, V1 and short PR interval, suggestive of a weak posteroseptal accessory pathway that manifests only at slow heart rates. This low-risk accessory pathway was deemed unlikely to cause tachyarrhythmia. 
Table 1 Relevant investigations

\begin{tabular}{|c|c|c|}
\hline Investigations & Results & Reference \\
\hline Fibrinogen $(\mathrm{g} / \mathrm{L})$ & 7.7 & $1.8-4.5$ \\
\hline D-dimer (ug/mL) & 2.55 & $<0.5$ \\
\hline $\operatorname{APTT}(\mathrm{s})$ & 38.9 & $27-37$ \\
\hline $\mathrm{PT}(\mathrm{s})$ & 14.3 & $11-14$ \\
\hline Factor II (\%) & 133 & $70-120$ \\
\hline Factor V (\%) & 134 & $70-120$ \\
\hline Factor VIII (\%) & 498 & $70-120$ \\
\hline Factor IX (\%) & 202 & $70-120$ \\
\hline Von Willebrand factor antigen (\%) & 371 & $56-160$ \\
\hline C-reactive protein (mg/L) & 200 & $0-5$ \\
\hline $\mathrm{ESR} \mathrm{mm} / \mathrm{h}$ & 70 & \\
\hline Lupus anticoagulant & Weakly positive & \\
\hline Anti-cardiolipin IgG (GPL units) & 35 & $0-20$ \\
\hline Anti-cardiolipin IgM (MPL units) & 28 & $0-20$ \\
\hline Anti-Sm, anti-RNP, anti-Jo 1, anti-SCL 70, anti-Ro, anti-La (RU/mL) & $<20$ & $0-20$ \\
\hline $\begin{array}{l}\text { Paraneoplastic antibody panel (anti-Hu, anti-Yo, Anti-Ri, anti-CV2, anti-amphiphysin, anti- } \\
\text { PNMA2/Ta, anti-recoverin, anti-SOX1, anti-titin, anti-Zic4, anti-GAD65, anti-Tr) }\end{array}$ & Negative & \\
\hline 24-h urinary noradrenaline (nmol/d) & 642 & $89-473$ \\
\hline 24-h urinary normetanephrine $(\mathrm{nmol} / \mathrm{d})$ & 5559 & $885-2880$ \\
\hline $\mathrm{ACTH}(\mathrm{pmol} / \mathrm{L})$ & 4 & \\
\hline 8 am cortisol (nmol/L) & 310 & $240-618$ \\
\hline \multicolumn{3}{|l|}{ Short Synacthen test $(\mathrm{nmol} / \mathrm{L})$} \\
\hline $0 \mathrm{~min}$ & 194 & \\
\hline $30 \mathrm{~min}$ & 537 & \\
\hline $60 \mathrm{~min}$ & 590 & \\
\hline \multicolumn{3}{|l|}{ Autonomic function test } \\
\hline & Blood pressure $(\mathrm{mmHg})$ & Heart rate $(/ \mathrm{min})$ \\
\hline Supine & $117 / 78$ & 70 \\
\hline Standing (3 min) & $125 / 89$ & 105 \\
\hline Supine & $118 / 78$ & 67 \\
\hline 60 -degree tilt (5 min) & $134 / 85$ & 98 \\
\hline Respiratory sinus arrhythmia & & $25 / \mathrm{min}$ \\
\hline Blood pressure before and after isometric exercise & $115 / 77 ; 128 / 87$ & \\
\hline Blood pressure before and after cold pressor stimulus & $115 / 76 ; 126 / 83$ & \\
\hline \multicolumn{3}{|l|}{ Lying to standing 30:15 ratio 1.31} \\
\hline \multicolumn{3}{|l|}{ Sympathetic skin response was present in palm and sole } \\
\hline The Valsalva maneuver could not be done because of COVID-19 infection control restriction & & \\
\hline
\end{tabular}

APTT activated partial thromboplastin time, $P T$ prothrombin time, ESR erythrocyte sedimentation rate

Transthoracic echocardiography was normal, and continuous heart monitoring did not detect any supraventricular tachycardia.

He was started on fludrocortisone and sodium chloride tablets empirically to raise intravascular volume and blood pressure, and consequently reduce reflex tachycardia. He also received pyridostigmine to improve gastrointestinal motility. Beta blockers were avoided because of concerns related to the accessory pathway. At discharge on day 43, his postural palpitations had improved. He was less disturbed by bouts of hyperhidrosis. At outpatient review on day 74, he did not complain of hyperhidrosis, and orthostatic tachycardia had resolved, albeit on medications. We suspect his illness was self-limiting, as these medications, including pyridostigmine, were not expected to have any significant effect on hyperhidrosis.

There was no diabetic retinopathy or other complications to suggest chronic poorly controlled DM. His HbA1c levels dropped from 8.8 to $7.6 \%$ over 3 weeks. However, he did not develop small-fiber neuropathy or maculopathy to suggest 
treatment-induced neuropathy of DM. Nevertheless, we cannot rule out hyperadrenergic autonomic neuropathy of nascent DM. We did not think he had Guillain-Barré syndromerelated dysautonomia. Nerve conduction study 2 weeks after onset of symptoms was normal; spinal tap was omitted, as he was taking warfarin. We also did a comprehensive screen for other causes of dysautonomia (Table 1). Mild anemia could have added to his symptoms, hemoglobin decreased from $12 \mathrm{~g} / \mathrm{dL}$ to about $10 \mathrm{~g} / \mathrm{dL}$; and we could not exclude physical deconditioning. This was our impression until his unelicited sudomotor complaints became apparent. He was a fit construction worker, and his pneumonia only required prone positioning and nasal oxygen supplementation. He was intubated for the embolectomy and extubated in a day. He was well hydrated, and was actively mobilized within 3 days of surgery by the physiotherapist. On the day of the autonomic function tests, he was walking and doing cycling exercises.

Our suspicion for COVID-19-related dysautonomia increased when we encountered three other COVID-19 patients with mild respiratory symptoms who developed acute near-vision difficulty from asymmetric accommodation deficiency. They had various pupillary abnormalities, namely Argyll-Robertson, inverse Argyll-Robertson and Adie's pupils. The patient with Adie's pupils lost his ankle and $\mathrm{H}$ reflexes, and developed orthostatic tachycardia (in press). We believe these cases highlight the occurrence of patchy dysautonomia in COVID-19 patients. We hypothesize that the autonomic dysfunction is similar to the post-infectious autonomic neuropathy seen in diphtheria. Accommodation difficulty, inverse Argyll-Robertson pupil, and delayed, mainly cardiovagal autonomic dysfunction and hyperhidrosis have been reported in diphtheria patients. In diphtheria, the pathology is post-infectious segmental demyelination [2-4]. The time course and immuno-thrombotic complication in our index patient also suggest post-infectious dysimmunity. Dysautonomia was reported in 21 out of 841 COVID-19 patients in the ALBACOVID registry [5]. No details of their autonomic dysfunction were given, but the authors also suggested dysimmunity involving the peripheral autonomic nervous system.

We considered hyperadrenergic postural orthostatic tachycardia syndrome (POTS) as an alternative hypothesis because the SARS-CoV 2 virus binds to angiotensin-converting enzyme 2 (ACE 2) receptors. Reduced ACE 2 activity, consequent increased angiotensin II and activation of renin-angiotensin-aldosterone, believed to underlie transient hypokalemia in some COVID-19 patients, may conceivably increase sympathetic activity to cause hyperadrenergic POTS. However, a hyperadrenergic state, although supported by urinary studies, does not explain all the manifestations in our patient.

In closing, we would like to raise awareness of COVID19 related dysautonomia.

Addendum

At second outpatient review, about 4 months after onset of COVID-19, the patient remained asymptomatic; he had no orthostatic tachycardia or hypotension. Lupus anticoagulant, anticardiolipin antibodies and urinary catecholamines had normalised. He had stopped the medications for about 2 months.

Author contributions TU, MQWP: Conceptualization, case evaluation and manuscript preparation. BEF, KFCL, JG, JYLT: Case evaluation and manuscript revision. The work was done at Tan Tock Seng Hospital, Singapore.

Funding None.

\section{Compliance with ethical standards}

Conflict of interest None of the authors have any conflicts of interest to disclose.

Ethical approval CIRB 2020/2014; A Survey of the Neurological Manifestations of COVID-19.

\section{References}

1. Iba T, Levy JH, Levi M et al (2020) Coagulopathy of coronavirus disease 2019. Critical Care Med Pub Ahead Print. https://doi. org/10.1097/CCM.0000000000004458

2. Fisher CM, Adams RD (1956) Diphtheritic polyneuritis; a pathological study. JNeuropathol Exp Neurol. 15:243-268. https://doi. org/10.1097/00005072-195607000-00002

3. Idiaquez J (1992) Autonomic dysfunction in diphtheritic neuropathy. J Neurol Neurosurg Psychiatry 55:159-161. https://doi. org/10.1136/jnnp.55.2.159

4. Piradov MA, Pirogov VN, Popova LM, Avdunina IA (2001) Diphtheritic polyneuropathy: clinical analysis of severe forms. Arch Neurol 58:1438. https://doi.org/10.1001/archneur.58.9.1438

5. Romero-Sánchez CM, Díaz-Maroto I, Fernández-Díaz E et al (2020) Neurologic manifestations in hospitalized patients with COVID-19: The ALBACOVID registry. Neurology. https://doi. org/10.1212/WNL.0000000000009937 